\title{
BMJ Open Electronic nicotine delivery system (ENDS) use during smoking cessation: a qualitative study of 40 Oklahoma quitline callers
}

\author{
Katrina A Vickerman, ${ }^{1}$ Laura A Beebe, ${ }^{2}$ Gillian L Schauer, ${ }^{3,4}$ Brooke Magnusson, ${ }^{1}$ \\ Brian A King ${ }^{4}$
}

To cite: Vickerman KA, Beebe LA, Schauer GL, et al. Electronic nicotine delivery system (ENDS) use during smoking cessation: a qualitative study of 40 Oklahoma quitline callers. BMJ Open 2017;7:e013079. doi:10.1136/bmjopen-2016013079

- Prepublication history for this paper is available online. To view these files please visit the journal online (http://dx.doi.org/10.1136/ bmjopen-2016-013079).

Received 23 June 2016 Revised 19 December 2016 Accepted 8 February 2017

\section{CrossMark}

\section{${ }^{1}$ Center for Wellbeing Research, Optum (formerly Alere Wellbeing, Inc.), Seattle, Washington, USA 2University of Oklahoma Health Sciences Center, Oklahoma City, Oklahoma, USA \\ ${ }^{3}$ Battelle Public Health Center for Tobacco Research, Battelle Memorial Institute, Seattle, Washington, USA ${ }^{4}$ Office on Smoking and Health Centers for Disease Control and Prevention, Atlanta, Georgia, USA}

Correspondence to Dr Katrina A Vickerman; Katrina.Vickerman@optum. com

\section{ABSTRACT}

Objectives: Approximately 10\% (40 000) of US quitline enrollees who smoke cigarettes report current use of electronic nicotine delivery systems (ENDS); however, little is known about callers' ENDS use. Our aim was to describe why and how quitline callers use ENDS, their beliefs about ENDS and the impact of ENDS use on callers' quit processes and use of FDAapproved cessation medications.

Design: Qualitative interviews conducted 1-month postregistration. Interviews were recorded, transcribed, double-coded and analysed to identify themes.

Setting: Oklahoma Tobacco Helpline.

Participants: 40 callers aged $\geq 18$ who were seeking help to quit smoking were using ENDS at registration and completed $\geq 1$ programme calls.

Results: At 1-month postregistration interview, 80\% of callers had smoked cigarettes in the last 7 days, almost two-thirds were using ENDS, and half were using cessation medications. Nearly all believed ENDS helped them quit or cut down on smoking; however, participants were split on whether they would recommend cessation medications, ENDS or both together for quitting. Confusion and misinformation about potential harms of ENDS and cessation medications were reported. Participants reported using ENDS in potentially adaptive ways (eg, using ENDS to cut down and nicotine replacement therapy to quit, and stepping down nicotine in ENDS to wean off ENDS after quitting) and maladaptive ways (eg, frequent automatic ENDS use, using ENDS in situations they did not previously smoke, cutting down on smoking using ENDS without a schedule or plan to quit), which could impact the likelihood of quitting smoking or continuing ENDS use.

Conclusions: These qualitative findings suggest quitline callers who use ENDS experience confusion and misinformation about ENDS and FDA-approved cessation medications. Callers also use ENDS in ways that may not facilitate quitting smoking. Opportunities exist for quitlines to educate ENDS users and help them create a coordinated plan most likely to result in completely quitting combustible tobacco.

\section{Strengths and limitations of this study}

- Dual users of cigarettes and electronic nicotine delivery system (ENDS) who were in the middle of an attempt to quit smoking were interviewed at 1-month postquitline registration, which allowed for timely reporting of quitting strategies and experiences using ENDS and cessation medications.

- In-depth qualitative interviews were completed with 40 dual users of cigarettes and ENDS to obtain detailed reports of experiences from a range of ENDS users.

- Interviews were recorded, transcribed and double coded to increase the trustworthiness of extracted themes.

- Findings from this small sample of Oklahoma Tobacco Helpline enrollees may not generalise to all ENDS users.

\section{INTRODUCTION}

Use of electronic nicotine delivery systems (ENDS), including electronic cigarettes (e-cigarettes), has increased considerably in recent years, particularly among current and former cigarette smokers. ${ }^{1}{ }^{2}$ In 2014, $47.6 \%$ of current cigarette smokers and $55.4 \%$ of recent former cigarette smokers had ever tried an e-cigarette. ${ }^{3}$ ENDS use among current smokers who call state quitlines is also common; state quitlines are free, typically phone-based tobacco cessation programmes available in all 50 states in the USA. ${ }^{4}$ In 2012, approximately one-third of quitline enrollees reported ever using ENDS, ${ }^{5}$ and from 2013 to $2015, \sim 10 \%$ reported current use at registration. ${ }^{6}$ An estimated half of these individuals reported using ENDS to try to quit smoking cigarettes. $^{56}$

Although ENDS may be commonly used by adults as a smoking cessation aid, they have not been approved by the US Food and 
Drug Administration (FDA) for this purpose. To date, there is no conclusive scientific evidence that ENDS are effective for long-term cessation from conventional cigarettes, ${ }^{7-11}$ and the long-term health impacts of ENDS use among adults are uncertain. ${ }^{8} 1213$ In light of this uncertainty, health professionals and treatment providers are seeking to identify the best way to help smokers who are using ENDS during their smoking cessation process. However, limited information is available about quitline callers' experiences using ENDS and their knowledge and beliefs about the products. This information is critical to help quitlines determine how best to address ENDS use given that an estimated 40000 ENDS users call quitlines each year for help quitting traditional tobacco products. ${ }^{4} 6$

Few studies have assessed factors related to ENDS use among US quitline callers. Accordingly, this study employed qualitative methods to assess quitline callers' opinions about and experiences using ENDS, including their beliefs about ENDS and smoking cessation, interactions with quitline counsellors about ENDS and perceptions of ENDS versus FDA-approved medications, including nicotine replacement therapy (NRT).

\section{METHODS}

\section{Participants}

Prenotification letters were mailed to 65 randomly selected participants who met study eligibility criteria $\sim 3$ weeks after Oklahoma Tobacco Helpline registration. Letters included a study overview and stated that study staff would be in contact about the study. Nineteen could not be reached after multiple attempts and six refused participation. Forty state quitline callers who wanted to quit smoking and called the Oklahoma Tobacco Helpline between November 2014 and February 2015 completed the interview. A minimum sample size target of 40 was selected to ensure inclusion of diverse ENDS users with regard to reasons for ENDS use. The interview team agreed that saturation was reached after 40 participants.

Participants were reached via phone, provided informed consent and completed an interview $\sim 1$ month after registration with the Helpline. Callers were eligible for the study if, at the time of registration, they (1) currently smoked conventional cigarettes; (2) were currently using ENDS as assessed by a standard quitline question ("Do you currently use electronic cigarettes, e-cigarettes or vapour cigarettes?"); (3) spoke English; (4) requested a Helpline intervention; (5) were 18 or older; (6) completed at least one call with a quit coach lasting at least $5 \mathrm{~min}$ and (7) provided consent for follow-up contact. Pregnant callers were excluded. Four participants used another form of tobacco product (eg, pipe, smokeless tobacco) in addition to cigarettes and ENDS at the time of registration. A US $\$ 49$ gift card to Amazon or Walmart was provided to participants who completed the interview. All study procedures were approved by the University of Oklahoma Institutional Review Board.

\section{The Helpline programme}

All participants were enrolled in a one-call or five-call phone-based cessation programme, which also included a 2-week or 8-week supply of NRT, a printed quit guide and access to an interactive online cessation resource. ${ }^{14}$ Intensity of Helpline service is determined by insurance status: Callers with no insurance are eligible for the five-call programme and 8-weeks of NRT, while callers with Medicaid also receive the five-call programme but only 2-weeks of NRT from the Helpline. Callers with private insurance are eligible for the single-call programme and 2 weeks of NRT. Coaching calls focus on developing a quit plan, building skills for coping with cravings and triggers, enlisting social support and using FDA-approved cessation medications. ${ }^{15} 16$

With regard to using ENDS to quit smoking, Helpline Quit Coaches were trained to not promote the use of ENDS for quitting tobacco and to provide education that there is not empirical evidence proving ENDS are safe and effective cessation tools. Smokers interested in switching to ENDS would not be discouraged from doing so and would be educated that the safety of longterm use of ENDS is unclear. ${ }^{17}$

\section{Interviews}

Interviews were conducted over the phone in private offices by two female graduate research assistants at the University of Oklahoma College of Public Health. Interviewers were enrolled in graduate training in epidemiology, had received extensive training in research methods, had interviewing experience from previous studies and received training in qualitative interviewing techniques. Interviewers followed a semistructured interview guide and were encouraged to ask additional probing questions. Topics discussed in the interviews were selected to investigate current gaps in knowledge about how ENDS were perceived and used by quitline callers. Topics included reasons for ENDS use, current ENDS and tobacco use, Helpline experience, experience with FDA-approved cessation medications, ENDS use details and intentions to quit. Interviews lasted $35.2 \mathrm{~min}$ on average (range: 22.1-61.5 min), were audio recorded and transcribed verbatim. A series of questions requesting feedback about the interview were also asked of the first eight participants.

\section{Coding and analysis}

Template analysis was used to guide the coding process. ${ }^{18}$ A preliminary codebook was developed after reading five transcripts. The codebook focused on topics queried during the interview and themes that arose in participants' responses. Themes were then further organised into larger concepts (eg, if initially separated topics yielded similar themes and were best grouped together), while grounding all identified themes in 
participant's words. Four individuals coded the interviews. Each interview was double-coded. Double-coding was reviewed, and consensus was reached for each interview. For analyses of coded data, our primary aim was to describe themes present in the data. We used a constant comparative approach, repeatedly returning to the transcripts and codes to ensure the accuracy and increase the depth of our summary of themes and to extract exemplary participant quotations. ${ }^{19}{ }^{20}$ Analyses were conducted in MAXQDA (software for qualitative data analysis, 1989-2016, VERBI Software-ConsultSozialforschung GmbH, Berlin, Germany).

\section{RESULTS}

\section{Participants}

Respondents were an average of 45 years old, $65 \%$ women, $73 \%$ white non-Hispanic, 68\% high school degree or less education and predominately low income $(65 \%<$ US\$25000 annual income) (table 1). Participants ranged in number of quit attempts prior to contacting the Helpline: for 6, their Helpline enrolment was a first quit attempt, 15 had one to two previous attempts, 10 had three to four previous attempts and 9 had five or more previous attempts. Thirty-three participants enrolled in the five-call programme and seven enrolled in a one-call programme. All received a 2-week or 8-week supply of NRT from the quitline. Participants completed 1.7 calls $(\mathrm{SD}=1.12)$ during the programme on average.

At registration, 19 reported using ENDS every day, with the remaining 21 participants reporting non-daily ENDS use; 33 reported using a tank system style ENDS (which is refilled with e-liquid by the user) and 37 said they were thinking about quitting ENDS in addition to cigarettes. All but one of the daily ENDS users used tank systems. About half reported they were primarily using ENDS to quit smoking, 13 said they were cutting down to quit smoking and 3 said they were only using ENDS to reduce smoking.

Table 2 displays products participants were using at the time of the qualitative interview. A total of $32 \mathrm{had}$ smoked cigarettes in the last 7 days, 25 were currently using ENDS and 21 were currently using an FDA-approved cessation aid. Twenty-nine of the participants were using two or three sources of nicotine (ie, cigarettes, ENDS and/or NRT).

\section{Interview themes}

How participants reported using ENDS and FDA-approved cessation medications

\section{Descriptions of ENDS use}

Participants discussed using ENDS as a quitting aid, as a bridge product when smoking was not allowed, and for a recreational experience. Callers also described using ENDS as a partial replacement for cigarettes-either as part of a cut down to quit strategy or, as a small number
Table 1 Demographics and tobacco use characteristics for respondents as reported at registration $(\mathrm{N}=40)$

\begin{tabular}{|c|c|c|}
\hline & $\mathbf{N}$ & Per cent \\
\hline Age, mean $\pm(S D)$ & \multicolumn{2}{|c|}{$45.0(14.1)$} \\
\hline Gender, female & 26 & 65.0 \\
\hline \multicolumn{3}{|l|}{ Education } \\
\hline Less than high school & 11 & 27.5 \\
\hline High school degree/GED & 16 & 40.0 \\
\hline $\begin{array}{l}\text { Some college/trade school or college/ } \\
\text { trade school degree }\end{array}$ & 13 & 32.5 \\
\hline \multicolumn{3}{|l|}{ Race/ethnicity } \\
\hline White, non-Hispanic & 29 & 72.5 \\
\hline \multicolumn{3}{|l|}{ non-Hispanic } \\
\hline $\begin{array}{l}\text { American Indian or Alaskan Native, } \\
\text { non-Hispanic }\end{array}$ & 5 & 12.5 \\
\hline Other race, non-Hispanic & 1 & 2.5 \\
\hline \multicolumn{3}{|l|}{ Health insurance status } \\
\hline Uninsured & 18 & 45.0 \\
\hline Medicaid & 8 & 20.0 \\
\hline Commercial & 8 & 20.0 \\
\hline Medicare & 5 & 12.5 \\
\hline 'Does not know' & 1 & 2.5 \\
\hline Employment status, employed & 19 & 47.5 \\
\hline Chronic condition, ${ }^{*}$ one or more & 21 & 52.5 \\
\hline Mental health condition, $\dagger$ one or more & 25 & 62.5 \\
\hline \multicolumn{3}{|l|}{ TTFU } \\
\hline Within 5 min & 17 & 42.5 \\
\hline $6-30 \mathrm{~min}$ & 14 & 35.0 \\
\hline More than $30 \mathrm{~min}$ & 6 & 15.0 \\
\hline Missing & 3 & 7.5 \\
\hline Cigarette use per day (cpd), mean $\pm(S D)$ & \multicolumn{2}{|c|}{$16.1(12.3)$} \\
\hline $\begin{array}{l}\text { Number of years used tobacco, 20+ } \\
\text { years }\end{array}$ & 28 & 70.0 \\
\hline \multicolumn{3}{|l|}{ Annual income } \\
\hline Less than US $\$ 10000$ per year & 18 & 45.0 \\
\hline US $\$ 10000-$ US $\$ 25000$ per year & 8 & 20.0 \\
\hline More than US\$25000 per year & 12 & 30.0 \\
\hline Missing & 2 & 5.0 \\
\hline \multicolumn{3}{|c|}{$\begin{array}{l}\text { *Chronic conditions included asthma, COPD, CAD and diabetes } \\
\text { (type II). } \\
\text { †Mental health conditions included ADHD, anxiety disorder, } \\
\text { bipolar, depression, gambling addiction, PTSD, schizophrenia and } \\
\text { substance use disorder. } \\
\text { ADHD, attention deficit hyperactivity disorder; CAD, coronary } \\
\text { artery disease; COPD, chronic obstructive pulmonary disease; } \\
\text { PTSD, post-traumatic stress disorder; TTFU, time to first use of } \\
\text { tobacco. }\end{array}$} \\
\hline
\end{tabular}

reported, using ENDS only for cutting down on cigarettes (not for quitting completely).

When asked whether ENDS helped them to quit or cut down on smoking, most participants believed ENDS helped them cut down on or quit cigarettes, including quitting completely $(n=7)$, quitting temporarily $(n=7)$ and cutting down without quitting $(\mathrm{n}=21)$. For example, one participant who quit temporarily and was using ENDS during her current quit attempt described:

To begin with, it [ENDS] took the place of the cigarette and I still got the nicotine...I totally didn't smoke for like 4 months. At that point, if I picked one [a cigarette] up 
Table 2 Current tobacco and ENDS use during qualitative interview at 1-month postregistration $(\mathrm{N}=40)$

N Per cent

\section{Current ENDS use}

Every day

1025.0

Some days

1537.0

Rarely

615.0

Not at all

922.5

Current tobacco use (not including ENDS)

Within the last 24 hours

2870.0

Within the last 7 days, but more than

410.0

24 hours ago

Within the last month, but more than

512.5

7 days ago

Within the last 3 months, but more than

25.0

1 month ago

Have you used NRT or cessation medications since

registration 1 month ago?

Yes

2870.0

No

1230.0

Current use of NRT or cessation

medications ${ }^{\star}$

Yes

2152.5

No

1947.5

Current dual use of products: cigarettes, ENDS and NRT or cessation medicationst

Cigarettes and ENDS $\quad 1025.0$

Cigarettes, ENDS and NRT/meds $\quad 922.5$

Cigarettes and NRT/meds $\quad 615.0$

Cigarettes only $\quad 717.5$

ENDS and NRT/meds $\quad 410.0$

ENDS only 25.0

NRT/meds only 25.0

*Participant reported currently using NRT or a cessation medication, or if current use was unclear, participant discussed recent use of NRT or cessation medication (ie, within the last 7 days)

tDefinitions of current use: any cigarette use in the last 7 days, current every day or some days ENDS use (excludes rarely and not at all), and current NRT/meds use defined as described in the above footnote.

ENDS, electronic nicotine delivery system; NRT, nicotine replacement therapy.

and tasted it, I didn't like the way it tasted. I didn't care for the light headed effect you got from it that comes with not smoking it. You don't smoke, that's the way a cigarette affects you...I have been [using cigarettes] for about the last month. We had some medical issues come up and a lot of stress and I just fell back into it, but [I'm] moving away from it. I'm doing the vapor stick more than the cigarettes...I have the patches, but I haven't started them.

Alternatively, one participant felt ENDS increased his urge to smoke and caused him to smoke more, and a small number reported that ENDS did not change their smoking. The latter participants noted that ENDS did not work for them because they were still smoking; one explained that carrying and maintaining their ENDS device were inconvenient.
When asked what they liked and what they did not like about using ENDS, participants reported positive and negative perceptions of ENDS use. However, overall, nearly all reported that they were satisfied with their ENDS device. Positive aspects of ENDS use included features of the use experience (eg, convenience of taking a puff quickly as desired vs lighting and finishing a cigarette; smell; taste; flavours), environmental factors (eg, no secondhand or thirdhand smoke, ash trays or lighted fire; can use where cannot smoke; less distracting to use while driving), positive health attributions or beliefs about ENDS (eg, health benefits such as breathing easier and less coughing, perceiving ENDS are healthier than cigarettes), beliefs that ENDS helped with quitting or cutting down (eg, behavioural substitute, control cravings, avoid weight gain), nicotine delivery and concentration options (eg, receiving needed nicotine; can taper nicotine or use no nicotine ENDS), cost of ENDS and others' reactions to ENDS use (others prefer ENDS, no judgement of use).

Negative perceptions of ENDS use were also reported, including features of the ENDS device (leaks, hard to maintain, weight/size/look, battery, breaks), beliefs that ENDS are harmful or potentially harmful to health, that ENDS do not help in quitting smoking (not satisfying, insufficient nicotine, does not help all of the time, increases urge to smoke), concerns about ENDS habit (addictive, continues hand to mouth habit), cost of ENDS (particularly to start) and the learning curve for use (process to find right device or learn to use, hard transition from smoking). Over a third of participants reported negative experiences or minor side effects, primarily coughing or sore throat/burning in back of throat. Individual participants reported headache, pain in lungs, dry mouth, dehydration and concern that ENDS worsened chronic obstructive pulmonary disease symptoms.

\section{Descriptions of FDA-approved cessation medication use}

When asked about past experiences using FDA-approved cessation medications, nearly all participants reported positive or mixed experiences. One participant described her positive experiences with cessation medications: "The patch is excellent because it takes the edge off." Other participants had mixed experiences. For example, a participant explained, "Chantix worked for me but sometimes [insurance] wouldn't pay for it and I can't afford it...My system on the patch-it rejects it." Only three participants reported that cessation medications were not perceived as helpful. For example, one participant described: "I have tried patches, the gum, the lozenges, and everything else and nothing has worked." Over half of the participants reported experiencing a side effect or reaction to an FDA-approved cessation medication, including bad dreams, skin reactions (rash, itchiness, burning sensation), emotional changes, upset stomach, sore or itchy throat, feeling jittery or having an abnormal taste in the mouth. 
A quarter of participants reported they were not using an FDA-approved cessation medication at the time of their interview because their quit date was still in the future and they were waiting to start NRT until their quit date. Some participants shared beliefs that they should not use patches and smoke; for example, "I put the patch on and I still smoked some...I would think, 'I'm just gonna have to take this patch off because you aren't supposed to smoke and wear the patch,' so I just took [the patch] off."

\section{Preferences for ENDS versus FDA-approved cessation medications for quitting smoking}

Participants were split on preferred methods for quitting smoking; similar numbers recommended an FDA-approved cessation medication only, ENDS only and using both at the same time. In addition, several participants reported no preference between ENDS and cessation medications. Several participants noted openness to trying any options that might work for them: "anyone [who] wants to quit smoking [should] try any method there is, because no person works the same."

Participants who recommended an FDA-approved cessation medication explained that medications helped with quitting or urges and had better nicotine delivery. For example, one participant described: "When I put it [patch] on, physically I don't crave a cigarette...that doesn't happen with the e-cigarette." Some participants also noted concerns about ENDS as a reason for their choice including believing ENDS are harmful or not knowing enough about safety, concerns about the addictiveness of ENDS or the hand to mouth habit, that using ENDS is not completely quitting, and insufficient nicotine delivery. One participant explained:

You're swapping out one cloud of smoke for another cloud of smoke [with ENDS]...You still have the habit of putting something in your mouth. You still have the habit of blowing something out of your mouth... What is the difference? I mean there's no evidence out there that proves that's [ENDS use] any better than actual smoking cigarettes...If you're gonna quit nicotine, quit nicotine all together. If you're trying to quit smoking, I guess you probably need to hit up on that nicotine patch.

Participants who felt ENDS were more helpful for quitting highlighted the importance of ENDS as a behavioural substitute for the hand to mouth habit of smoking. They felt ENDS provided a better transition from smoking, believed ENDS helped avoid weight gain during quitting, liked that they could use ENDS as needed, liked the throat hit from ENDS and preferred the option to step down on nicotine. For example, one participant shared,

It [patch] was a lot harder because you have the patch [and] there is [a] no smoking period... The hand to mouth gesture is there, it's integrated in your system I think, if you smoke for a long time. So it would be a lot harder to go from smoking a cigarette to having the patch on your arm. That's really hard to do. In my thought process, vaping to quit will actually be a better thing because there's still that interaction between hand to mouth versus food going hand to mouth.

Most of these participants noted side effects with FDA-approved cessation medications or that cessation medications did not work well for them in the past or did not provide sufficient nicotine. Finally, participants who believed it was most helpful to use FDA-approved cessation medications and ENDS together for quitting provided several explanations. Some noted this approach would be preferred especially for long-time smokers, some felt ENDS helped them with quitting more than cessation medications and some felt an FDA-approved cessation medication helped them more with quitting than the ENDS product. Some in this group of participants recommended using patches and then ENDS (with or without nicotine) as a stopgap for relapse or when urges were high, noting that patches alone were not enough.

\section{Misinformation and confusion about relative harm of ENDS and FDA-approved medication \\ Views on relative harm of products}

Participants were asked to rank ENDS, NRT and cigarettes from most to least harmful and explain their rankings. Slightly more than half of the participants ranked FDA-approved cessation medications as least harmful, just less than half ranked ENDS as least harmful and a few felt FDA-approved cessation medications and ENDS were equally harmful. All ranked cigarettes as most harmful. Some participants ranked FDA-approved cessation medications as more harmful than ENDS due to their reactions to FDA-approved cessation medications. For example, one participant explained, "I would put [patches] in the rank close to the cigarette, [because they] make your heart beat really fast." Some participants provided explanations for their ranking choices that involved misinformation about ENDS, FDA-approved cessation medications or nicotine, as described in the next section. Several participants also expressed lack of confidence in their knowledge about the relative harm of products, for example, stating, "I would probably have to say that I don't know. I think the vapor's more harmful. I really do not know."

\section{Misinformation and confusion about ENDS}

Participants reported several inaccurate beliefs about the safety and constituents of ENDS. Some participants expressed beliefs that ENDS and the aerosol ENDS produce are completely safe. For example, one participant said, "There's not any chemicals in [ENDS] like there are in actual cigarettes," and another stated, "It's a vapor, I mean, you're not really hurting anybody. You could smoke it inside the building." One participant reported believing she was not receiving any nicotine 
from ENDS; she explained, "[When] I use the ecigarette, I really don't think I am getting the nicotine or anything in my lungs because it evaporates. I think I am just getting a taste of nicotine."

\section{Views on long-term ENDS use}

When asked what they thought about long-term use of ENDS, almost equal numbers were concerned, supportive and unsure. Some participants' explanations reflected confusion or misbeliefs about ENDS. Participants who had concerns about long-term ENDS use either believed that ENDS are harmful or detrimental to health or thought ENDS may have negative impacts if used long-term, but often thought short-term use was likely okay. Those who were unsure often saw benefits to ENDS use (not smoking, feeling healthier) or had not personally experienced any negative impacts of using ENDS, but either (1) expressed uncertainty about long-term use due to unknown constituents of ENDS, the potential impact of aerosol on lungs or using an addictive product or (2) did not know what to think about long-term use (ie, did not know because they had not read or heard anything about it or believed there are conflicting opinions on this subject). Participants who said they were supportive of long-term use were most likely to report inaccurate information about ENDS, such as that ENDS and the aerosol it produces are completely safe (ie, the aerosol is just water vapour) or that there were no concerns as long as one used ENDS products that did not contain nicotine. Some of these participants also noted the relative benefit of using ENDS rather than cigarettes.

\section{Misinformation about FDA-approved cessation medications}

Some participants also made statements that reflected concerns about the safety of NRT or how to use NRT. For example, one participant stated, "The patch has definitely got tar in it." Another participant who believed the patch and ENDS were equally harmful explained, "with the patch, the nicotine [is] being absorbed into your skin-that is chemicals, so that is not good." A third participant who rated patches as more harmful than ENDS shared, "I don't really know how to explain it...it's just that I'm scared to use them [patches]." Another participant ranked NRT as more harmful than ENDS because of concerns about overdosing on NRT.

\section{Concerns about nicotine}

Some participants focused on the safety of nicotine regardless of whether it was delivered from ENDS or FDA-approved cessation medications. One participant stated, "Nicotine in pure form causes Alzheimer's." Two participants believed their $0 \mathrm{mg}$ nicotine ENDS product was the least harmful product, "because nicotine is bad for you;" both participants were still smoking cigarettes at the time of the interview. Several others were also unsure whether ENDS or NRT are more harmful or ranked NRT as more harmful because they viewed nicotine dependence or use as the main concern. Several ranked ENDS as less harmful than the nicotine patch because they believed ENDS deliver less nicotine.

\section{Strategies used for incorporating ENDS in smoking cessation plans}

Participants described how they used ENDS devices during their smoking cessation process. We categorised these behaviours into potentially adaptive and maladaptive quit behaviours. These categories were not mutually exclusive; some participants reported behaviours that may be less likely to harm their quit process or possibly help with quitting (potentially adaptive) as well as behaviours that may be more likely to establish a separate ENDS habit or were less likely to reduce smoking (potentially maladaptive).

\section{Potentially adaptive quit behaviours}

On the basis of the participants' descriptions for how they incorporated ENDS into their smoking cessation plan, we identified five examples of potentially adaptive behaviours. Some participants were using ENDS only, but decided to or planned to switch to using a patch along with a no-nicotine ENDS: "I'm trying something different now that I got my patches in. I'm going to use patches for the nicotine. And then I'm going to fill my e-cig with the zero nicotine. That way I have the habit still and I'm getting the nicotine, but I can cut back with the patches." Second, other participants used NRT, such as the patch, and used ENDS with nicotine when cravings were high to avoid picking up a cigarette. Third, multiple participants discussed stepping down the milligrams of nicotine in their ENDS liquid to wean off nicotine. For example, one participant described, "It [ENDS] took the place of the cigarette and I still got the nicotine and you're able to cut down the amount of nicotine you get in the vapor. You can get it in a 24, 18, $12,7,2$, or $0[\mathrm{mg} / \mathrm{ml}$ nicotine concentration $]$ and I just stepped down." Fourth, several participants used ENDS to cut down and completely replace smoking cigarettes and then switched to the patch to quit cigarettes and ENDS. Finally, a small number of participants completely switched from cigarettes to ENDS or cut down cigarettes by replacing with ENDS and eventually fully replaced all cigarettes with ENDS.

\section{Potentially maladaptive quit behaviours}

Four types of potentially maladaptive behaviours for incorporating ENDS into smoking cessation plans were identified. First, more than half of the participants reported using ENDS in situations where they did not or could not smoke, most frequently at work, in their car, at home or in stores. Second, a group of participants had difficulty describing their ENDS use because it had become a frequent, automatic behaviour. One participant explained, "I can't even count to be honest with you... I just go 'oh look, I want this' and I grab it. I have a lanyard that's around my neck all day long." Third, 
several participants who were cutting down to quit, described partially replacing cigarettes with ENDS, but choosing to smoke a cigarette when they were stressed or their cravings were high. Fourth, while some participants described a plan for reducing nicotine intake and weaning off ENDS, others were cutting down to quit without a plan or schedule.

\section{What is the role of the quitline?}

ENDS users were asked what they remembered from their conversation with Quit Coaches about ENDS. Half remembered some conversation with their Quit Coach about ENDS. The top four messages participants reported receiving from Quit Coaches were: concerns about ENDS, much is unknown about ENDS, use encouraged or 'ENDS are okay' and avoiding dual use of ENDS and cigarettes. One participant described how use was discouraged, "I told them I used vapors and they informed me that there [were] no FDA regulations on it and they suggested I didn't use those at all." Conversations about ENDS with Quit Coaches had varying impact for different participants. The aforementioned participant described that she felt the quitline was "very much discouraging use...I blew it off...to each their own on their opinions on vaping. I don't see anything wrong with vaping." However, another participant stated that her conversation with the Quit Coach changed her view of ENDS, "He made a really, really good point that they are not regulated and you don't know what is in it. He really put an idea in my head." Several participants reported action or belief changes following their conversation with a coach.

Participants were also asked what other messages they would like from the quitline about ENDS. The majority had no response or did not know. Several requested more information in general or about the safety of ENDS. A few participants had hoped to hear that it was a good idea to vape or that ENDS are helpful with quitting. Another participant described that she assumed she should stop using ENDS before starting NRT because the quitline recommended "that you discontinue all nicotine intake other than the lozenges and the gum," but an explicit conversation about guidance on using ENDS and patches would have been helpful. Overall, $\sim$ three-quarters of the 40 participants said they would recommend other ENDS users call the quitline.

\section{DISCUSSION}

Analysis of interviews with 40 quitline callers who were using ENDS during an attempt to quit cigarette smoking revealed themes that may be relevant to consider when approaching treatment with dual users of cigarettes and ENDS. ENDS products were used in multiple ways, including as a cessation aid, a partial replacement for smoking prior to quitting, a bridge product when unable to smoke and an enjoyable experience. In contrast, FDA-approved cessation medications were typically discussed only in the context of cessation, highlighting the added complexity of counselling smokers using ENDS. This contrast likely contributes to NRT and ENDS being used differently. For example, some participants discussed waiting to use NRT until their quit date or stopping NRT use if they relapsed to smoking; these use 'rules' that were not discussed for ENDS. The majority of participants had positive use experiences with ENDS, as well as with some FDA-approved cessation medications. However, participants were split on whether FDA-approved cessation medications, ENDS or using FDA-approved cessation medications and ENDS simultaneously worked best for them for quitting smoking.

In addition, some participants shared inaccurate beliefs about ENDS, FDA-approved cessation medications and nicotine. Participants' beliefs about the products, whether accurate or inaccurate, can likely impact how and whether participants incorporate ENDS and FDA-approved cessation medications into their quit plan. Findings from these interviews suggested that state quitline callers who use ENDS would benefit from additional education about ENDS, FDA-approved cessation medications, nicotine and the relative harm of these products. Specifically, quitlines and other health professionals have an opportunity to provide information about ENDS and quitting that will maximise the likelihood of helping individual smokers quit completely. Information callers receive may also impact what information they share with others about ENDS and their use of ENDS around others, including children.

The effectiveness of ENDS use for promoting longterm cessation from conventional cigarettes is uncertain. ${ }^{7-11}$ However, many smokers report using ENDS to help them quit smoking, ${ }^{5621}$ and some former smokers report successfully using ENDS to quit. ${ }^{22-28}$ On the basis of principles of behaviour change, strategies were noted by respondents in this study that may be less likely to result in harm during a quit process (potentially adaptive behaviours), including using patches and ENDS together similar to combination therapy, using ENDS to cut down and FDA-approved cessation devices to quit completely and fully replacing cigarettes with ENDS. Potentially maladaptive behaviours of cutting down using ENDS were also noted, including using ENDS in places they did not previously smoke, using ENDS in a frequent unplanned manner and cutting down to quit without a plan or schedule. ENDS use in situations participants did not previously smoke may serve to increase nicotine dependence, undermine the impact of tobacco free policies, expose others to secondhand aerosol and establish dual use of ENDS and cigarettes. Smokers who cut down to quit, but continue to smoke in high craving situations, may not develop coping skills for stress or cravings in difficult situations and may experience the remaining cigarettes they smoke as particularly reinforcing. Finally, research has shown that cutting down to quit without a plan or schedule is less likely to be effective. $^{29}{ }^{30}$ These findings suggest that treatment providers 
may find it useful to identify and discuss maladaptive behaviours during smoking cessation treatment. Future research is also warranted to better understand how ENDS and cessation medication are used together during a quit attempt, as well as the effectiveness of these strategies.

Previous studies suggest that ENDS experimentation or use may help to motivate a quit attempt (MAXQDA, software for qualitative data analysis, 1989-2016).$^{10-22}$ 24-32 It is possible that some smokers who try ENDS will have a positive experience that may bolster their motivation and intention to quit (eg, feeling healthier, managing cravings, reducing cigarettes per day), which appeared to be the case for some in this study. Although research has shown that reducing cigarette consumption is not sufficient to fully reduce health risks of smoking, ${ }^{23} 3334$ cigarette reduction may be a step towards quitting for individuals who have been unable to stop abruptly. Some research suggests that a cut-down-to-quit strategy that is guided on a schedule may yield similar outcomes as abrupt quitting; ${ }^{29} 30{ }^{35}$ however, cutting down to quit has been found to be less effective in real-world samples. ${ }^{36}$ In the present study, it was uncertain how aware participants were of the importance of completely quitting combustible tobacco. Given the number using a cut-down-to-quit strategy, this represents an important area for future research. Additionally, varying reasons and methods for using ENDS, varying experiences with using ENDS while quitting smoking and changes in ENDS use from registration to the 1 month interview highlight that the concept of 'dual use' of ENDS and cigarettes is not unidimensional. Future research is important to examine how patterns of dual use change over time, particularly during different phases of a smoker's quit process, taking into account current reasons for use. For example, using ENDS to prevent relapse with some brief smoking lapses may need to be viewed differently than long-term use of ENDS to cut down on smoking with no plans to quit smoking completely.

This study is subject to at least five limitations. First, the findings from this small sample may not generalise to all ENDS users. Second, the sample may also include more ENDS 'treatment failures' than in the general population; if smokers had successfully quit using their ENDS device, they could have been less likely to call the quitline for assistance. ${ }^{7}$ Third, most callers had completed one quitline call, and some had a limited memory of the call. Interviewing callers after they had an opportunity to complete more calls could yield different information. Fourth, it was not possible to fully account for the type of ENDS device used, use topography or proficiency/experience as a user. Given that these characteristics can impact nicotine delivery, ${ }^{37} 38$ these factors may have influenced preferences for use of ENDS versus FDA-approved cessation medications for quitting smoking. Finally, it was unclear if timing of the interviews (eg, New Year and holidays) may have led to delayed quit dates, or if that was part of the quitting process for individuals in this sample.

In conclusion, in this sample of 40 ENDS users who contacted a state quitline for help quitting smoking, some ENDS users had confusion or misinformation about ENDS, FDA-approved cessation medications, nicotine and the relative harm of these products. ENDS were being used in ways that were unlikely to help with quitting smoking, as well as in ways that may potentially facilitate quitting smoking. These findings suggest that quitlines have a unique opportunity to educate a significant number of ENDS users and to help them create a coordinated quit plan most likely to result in completely quitting combustible tobacco. These findings also suggest important avenues for future research. Strategies for educating smokers about ENDS and, if necessary, changing inaccurate beliefs about products warrant development and testing. Additionally, future research could examine whether the use of cut-down-to-quit strategies and relapse experiences are different for individuals who use ENDS during their quit process. Finally, for quitline callers who plan to use ENDS as part of their quit attempt, research is warranted to determine whether certain information about ENDS or behavioural support from the quitline may improve callers' success with quitting smoking.

Acknowledgements The authors thank and acknowledge Erica Salmon and Kristina Muramoto for coding interviews; Lacy Brame, MS, and Dana Mowls, $\mathrm{MPH}$, for conducting the participant interviews and Susan Zbikowski, PhD, and Ann Malarcher, PhD, for input on the study design.

Contributors KAV, LAB, GLS and BM contributed to the interview guide and coding methodology. LAB oversaw participant interviews. KAV and BM (with two other coders) coded interviews. KAV led the analysis of coded data, performed the literature review and took primary responsibility for writing the paper. All authors contributed to the conceptualisation of the study, study design, interpretation of data and the preparation of the manuscript. All authors approved the final version of the manuscript.

Funding This project was funded in part by Centers for Disease Control \& Prevention Contract \#200-2014-M-60619 and the Oklahoma Tobacco Settlement Endowment Trust.

Disclaimer The findings and conclusions in this report are those of the authors and do not necessarily represent the official views of the Centers for Disease Control and Prevention.

Competing interests All authors have completed the ICMJE uniform disclosure form at http://www.icmje.org/coi_disclosure.pdf and declare: KAV and BM report funding from Centers for Disease Control and Prevention during the conduct of the study and are employees of Optum (formerly Alere Wellbeing, Inc.), the provider of quitline services for participants in this study; LAB reports grants from Oklahoma Tobacco Settlement Endowment Trust during the conduct of the study.

Ethics approval University of Oklahoma Institutional Review Board.

Provenance and peer review Not commissioned; externally peer reviewed.

Data sharing statement Data sharing: no additional data are available.

Open Access This is an Open Access article distributed in accordance with the Creative Commons Attribution Non Commercial (CC BY-NC 4.0) license, which permits others to distribute, remix, adapt, build upon this work noncommercially, and license their derivative works on different terms, provided the original work is properly cited and the use is non-commercial. See: http:// creativecommons.org/licenses/by-nc/4.0/ 


\section{REFERENCES}

1. Regan AK, Promoff G, Dube SR, et al. Electronic nicotine delivery systems: adult use and awareness of the 'e-cigarette' in the USA. Tob Control 2013;22:19-23.

2. King BA, Patel R, Nguyen $\mathrm{KH}$, et al. Trends in awareness and use of electronic cigarettes among US adults, 2010-2013. Nicotine Tob Res 2015;17:219-27.

3. Schoenborn MPH, Gindi RM. Electronic cigarette use among adults United States, 2014. NCHS Data Brief 2015;217:1-8.

4. North American Quitline Consortium (NAQC) (2015). Results from the 2013 NAQC Annual Survey of Quitlines Webinar Presentation, North American QuitlineConsortium (NAQC). Phoenix, AZ, USA, 2013. http://www.naquitline.org/?page=2013Survey (accessed $23 \mathrm{Ju}$ 2015).

5. Vickerman KA, Carpenter KM, Altman T, et al. Use of electronic cigarettes among state tobacco cessation quitline callers. Nicotine Tob Res 2013;15:1787-91.

6. Vickerman KA, Beebe L, Schauer G, et al. ENDS users who call quitlines: surveillance data and research findings. 2015 Annual North American Quitline Consortium (NAQC) Conference; Atlanta, GA, 2015.

7. McRobbie H, Bullen C, Hartmann-Boyce J, et al. Electronic cigarettes for smoking cessation and reduction. Cochrane Database Syst Rev 2014;12:CD010216.

8. Grana R, Benowitz N, Glantz SA. E-cigarettes: a scientific review. Circulation 2014;129:1972-86.

9. Bullen C, Howe C, Laugesen M, et al. Electronic cigarettes for smoking cessation: a randomised controlled trial. Lancet 2013;382:1629-37.

10. Caponnetto P, Campagna D, Cibella F, et al. EffiCiency and Safety of an eLectronic cigAreTte (ECLAT) as tobacco cigarettes substitute: a prospective 12-month randomized control design study. PLoS One 2013;8:e66317.

11. Bernstein SL. Electronic cigarettes: more light, less heat needed. Lancet Respir Med 2016;4:85-7.

12. Farsilinos KE, Kistler KA, Gillman G, et al. Evaluation of electronic cigarette liquids and aerosol for the presence of selected inhalation toxins. Nicotine Tob Res 2015;17:168-74.

13. Fagerstrom K, Etter JF, Unger JB. E-cigarettes: a disruptive technology that revolutionizes our field? Nicotine Tob Res 2015;17:125-6.

14. Nash CM, Vickerman KA, Kellogg ES, et al. Utilization of a web-based vs integrated phone/web cessation program among 140,000 tobacco users: an evaluation across 10 free state quitlines. J Med Internet Res 2015;17:e36.

15. Bandura A. Social cognitive theory. In: Vasta R. ed. Annals of child development, six theories of child development. Vol 6. Greenwich (CT): JAI Press; 2015:1-60.

16. US PHS Guidelines. Treating tobacco use and dependence: 2008 update. Rockville (MD): US Department of Health and Human Services; Public Health Services, 2008

17. Alere Wellbeing, Inc. (2014) Alere position statement on E-cigarettes. Seattle, WA.

18. King N. Using templates in the thematic analysis of text. In: Cassell $\mathrm{C}$, Symon G, eds. Essential guide to qualitative methods in organization research. London: Sage, 2004:256-70.

19. Patton MQ. Qualitative research \& evaluation methods. 3rd edn. Thousand Oaks (CA): Sage, 2001.
20. Sandelowski M. Whatever happened to qualitative description? Res Nurs Health 2000;23:334-40.

21. Brown J, West R, Beard E, et al. Prevalence and characteristics of e-cigarette users in Great Britain: findings from a general population survey of smokers. Addict Behav 2014;39:1120-5.

22. Barbeau AM, Burda J, Siegel M. Perceived efficacy of e-cigarettes versus nicotine replacement therapy among successful e-cigarette users: a qualitative approach. Addict Sci Clin Pract 2013;8:5.

23. Bjartveit $K$, Tverdal A. Health consequences of smoking 1-4 cigarettes per day. Tob Control 2005;14:315-20.

24. Etter JF, Bullen C. Electronic cigarette: users profile, utilization, satisfaction and perceived efficacy. Addiction 2011;106:2017-28.

25. Etter JF, Bullen C. A longitudinal study of electronic cigarette users. Addict Behav 2014;39:491-4.

26. Foulds J, Veldheer S, Berg A. Electronic cigarettes (e-cigs): views of aficionados and clinical/public health perspectives. Int J Clin Pract 2011;65:1037-42.

27. Siegel MB, Tanwar KL, Wood KS. Electronic cigarettes as a smoking-cessation: tool results from an online survey. Am J Prev Med 2011;40:472-5.

28. Dawkins L, Turner J, Roberts A, et al. 'Vaping' profiles and preferences: an online survey of electronic cigarette users. Addiction 2013;108:1115-25

29. Lindson-Hawley N, Aveyard P, Hughes JR. Reduction versus abrupt cessation in smokers who want to quit. Cochrane Database Syst Rev 2012;11:CD008033.

30. Cinciripini PM, Lapitsky L, Seay S, et al. The effects of smoking schedules on cessation outcome: can we improve on common methods of gradual and abrupt nicotine withdrawal? J Consult Clin Psychol 1995;63:388-99.

31. Etter JF. Electronic cigarettes: a survey of users. BMC Public Health 2010;10:231.

32. Wagener TL, Meier E, Hale JJ, et al. Pilot investigation of changes in readiness and confidence to quit smoking after e-cigarette experimentation and 1 week of use. Nicotine Tob Res 2014;16:108-14.

33. USDHHS. The health consequences of smoking-50 years of progress: a report of the surgeon general. Atlanta (GA): US Department of Health and Human Services, Centers for Disease Control and Prevention, National Center for Chronic Disease Prevention and Health Promotion, Office on Smoking and Health, 2014.

34. Tverdal A, Bjartveit K. Health consequences of reduced daily cigarette consumption. Tob Control 2006;15:472-80.

35. Beard E, McNeill A, Aveyard P, et al. Association between use of nicotine replacement therapy for harm reduction and smoking cessation: a prospective study of English smokers. Tob Control 2013;22:118-22.

36. Schauer GL, Malarcher AM, Babb SD. Gradual reduction of cigarette consumption as a cessation strategy: prevalence, correlates, and relationship with quitting. Nicotine Tob Res 2015;17:530-8.

37. Talih S, Balhas Z, Eissenberg T, et al. Effects of user puff topography, device voltage, and liquid nicotine concentration on electronic cigarette nicotine yield: measurements and model predictions. Nicotine Tob Res 2015;17:150-7.

38. Davis B, Dang M, Kim J, et al. Nicotine concentrations in electronic cigarette refill and do-it-yourself fluids. Nicotine Tob Res 2015;17:134-41. 